\title{
Nestmate recognition in social insects is sometimes more complex than an individual based decision to accept or reject
}

\author{
Brian R. Johnson • Ellen van Wilgenburg • \\ Neil D. Tsutsui
}

Received: 16 November 2011 /Revised: 21 November 2011 / Accepted: 29 November 2011 /Published online: 10 December 2011

(C) Springer-Verlag 2011

Early work on the setting of acceptance thresholds for nestmate recognition suggested that an overlap between the odor templates used by different colonies could lead to a tradeoff between accepting nestmates and rejecting nonnestmates (Reeve 1989). In a recent paper, we pointed out an alternative approach to this problem (Johnson et al. 2011). We started with a literature review that suggests that rejection of nestmates is exceedingly rare in ants, although it does seem to occur in bees and wasps. Hence, ants do not seem to need to reject their own nestmates in order to exclude non-nestmates. Following this literature review, we suggested that if social insects are using gestalt mechanisms to generate a template of acceptable odors (reviewed by Tsutsui 2004; Martin and Drijfhout 2009), then the concept of overlap between the cues used by different colonies might be of little utility. The relevant question is rather one of errors due to limited sensory acuity. We then interpreted the rejection errors of bees from this perspective and showed that per encounter rejection rates may be part of a more accurate colony level collective decision-making

Communicated by J. Traniello

B. R. Johnson $(\square)$

Department of Entomology, University of California, Davis,

1 Shields Avenue,

Davis, CA 95616, USA

e-mail: brnjohnson@ucdavis.edu

E. van Wilgenburg

Department of Zoology, University of Melbourne,

Melbourne, Victoria 3010, Australia

\section{N. D. Tsutsui}

Department of Environmental Science, Policy and Management,

University of California Berkeley,

130 Mulford Hall, \#3114,

Berkeley, CA 94720-3114, USA process. Essentially, per encounter rejection rates can amplify over many encounters to a more accurate level of recognition than would be the case for a single interaction. Couvillon et al. (2011), in their commentary on this work, argue that our results are based on false assumptions. We disagree. Couvillon et al. raise three issues with our work, to which we respond below.

Couvillon et al. (2011) argue that we did not consider previous work on individual-level responses by guards (Couvillon et al. 2008). They further suggest that this work on individual-level responses undermines our model. We recognize that individual social insects can change their behaviors in response to threats, and we have recently published research on exactly this topic (van Wilgenburg et al. 2010). However, the role of collective decision-making has been largely overlooked in the field of nestmate recognition, and the explicit goal of Johnson et al. was to explore the nature and efficacy of such systems. Ultimately, the stringency of nestmate recognition is likely regulated by both individual-level changes and collective responses, as the two are not mutually exclusive.

Nevertheless, the study highlighted by Couvillon et al. (2008) suffers from a number of shortcomings, rendering its overall conclusions about honey bee nestmate recognition dubious. In their experiment, Couvillon et al. (2008) put a feeder dish in the entrance of one colony in an apiary under the assumption that this will cause that colony to go out and rob its neighbors. They inform us that they subsequently recorded a 10-fold increase in fights at the entrances of their study colonies along with a $20 \%$ increase in guard numbers. They then point out that the overall increase in defensiveness that they recorded was completely accounted for by an individual level response by guards who decreased their acceptance rates. What Couvillon et al. have not mentioned in their commentary are the actual numbers involved. The 
number of guards in their experiment went from 1.9 to 2.3, while the number of fights went from 0.005 per guard to 0.06 . The problem here is that a 10 -fold increase from a very small number is still a very small number. If we contrast this experiment with the classic exploration of robbing behavior conducted by Butler and Free (1952), we can see the problem with the Couvillon et al. approach. Butler and Free released large numbers of foreign bees into the entrances of several colonies one at a time and recorded the colony level response. What they found was a nonlinear response such that the colony initially was not defensive until a threshold number (often in the hundreds) of invaders was reached. At this point, the colony became alerted and large numbers of guards rushed to the entrance. Before the rise in guard numbers, few of the introduced foreign bees were mauled by guards, whereas after the introduction of sufficient foreign bees, nearly all the introduced foreign bees were mauled. An additional finding of this study was that bees exhibit different behaviors when they are mauled by guards. Bees entering their own nest submit to mauling without trying to escape. Mauling can last for several minutes with the bee usually attaining entry to the nest. Bees that submit to mauling are not killed, and so presumably can try again to gain entry if they are evicted from the entrance. Bees that are robbing another nest, however, try to escape from the guards, and when they cannot, they fight back leading to the death of one of the bees. Bees that are trying to gain entry to a foreign nest, in fact, exhibit several forms of evasive behaviors, which alone are sufficient for guards to recognize them as invaders. The reader is encouraged to read this study, as the relevant natural history is quite involved and there is not sufficient space here to do it justice.

If we consider Couvillon et al.'s study in light of the natural history of nest defense in honey bees, we see several problems. First, their attempt to trigger a defensive response by feeding one colony sugar syrup only partially worked. They triggered a minor increase in defensiveness, which was sufficient to generate an individual-level response, but not strong enough to trigger a full colony level defensive response. The reason their recorded increase in defensiveness was accounted for by the individual level response is therefore because there was almost no colony-level response. The second problem has to do with a common sense consideration of the numbers involved. If a colony posts three guards, the most they can do when an attack occurs (attacks can be strong enough to lead to the death of weak colonies) is lower their acceptance threshold to its minimum and work at their highest rate. In other words, they can reject up to three bees at a time. This is because rejecting, which involves mauling, is time consuming. Moreover, if one considers that guards must also inspect numerous nestmates, and that rejected robbers have a high probability of killing a guard, then it is clear that a group level response in which more guards are recruited is necessary.

The third, and most pressing, problem with Couvillon et al.'s approach is that they measured aggression rather than rejection. As Butler and Free (1952) showed, aggression against bees that will eventually be accepted is part of nestmate recognition in honey bees. Hence, categorizing any bee that receives aggression as rejected, as Couvillon et al. (2008) did, ignores the natural history of this system. To accurately measure acceptance, the final outcome (entry or eviction) must be recorded. Furthermore, since so much of a guard's decision to reject is based on behavioral interactions, the bees used in experiments cannot be disoriented due to chilling or any other treatment that precludes their ability to respond normally when accosted by guards.

In conclusion, Couvillon et al. (2011) accuse us of ignoring their work on individual-level responses. This is not the case. We chose to model that aspect of the process that is novel. If we had included both an individual level response and a group response, then the role of the group response would have been less clear. Our model is certainly a simplification of the real process, which includes an important individual-level response. Nevertheless, adding an individuallevel response to the model would make the bees better at nestmate recognition. The individual level response does not invalidate the importance of the group level response. The group level response, in fact, could work without the individual level response (though less efficiently). The individual-level response, in contrast, cannot possibly account for nest defense alone, since a small number of guards cannot defend against a much larger group, no matter how much they reduce their acceptance thresholds.

The second issued raised by Couvillon et al. is that if guard errors are not independent (as they are in the model), then multiple interactions between guards and nonnestmates cannot amplify the overall probability of rejection. In general, we view it as an oversimplification to either assume that the rejection behaviors displayed by different individuals are wholly autocorrelated, or to assume that they are wholly independent of each other. Both rejection and aggression probabilities will be determined by the composition of recognition labels and the development of recognition templates, but these may vary through space and time (as we discuss in the original paper). Moreover, myriad other factors (age, experience, health, etc.) can influence a worker's decision to accept or reject another and, to the extent that these factors vary among workers, they will display variation in rejection probabilities.

However, the above conceptual response is secondary to how we view this criticism. Our primary response is that aggression is not equivalent to rejection in honey bees, so the numbers Couvillon et al. recorded do not reflect overall rejection rates. Furthermore, for argument's sake, consider 
what would be true if Couvillon et al. are correct on this point. Essentially, they are saying that all guards are likely to make the same errors and hence, per encounter rejection rates are also overall rejection rates. Hence, if one records a $20 \%$ per encounter rejection rate of nestmates (as these authors did), then this would mean that honey bee colonies evict (essentially kill) $20 \%$ of their own nestmates. In some colonies, Couvillon et al. (2008) recorded over 50\% per encounter rejection rates. We doubt any colony could survive such a disastrous inability to recognize self.

Couvillon et al. (2011) raise three points as part of their last criticism. First, they argue that the adaptive shift in acceptance threshold model that they are testing is predicted to occur within species only, and no pattern is expected across species. This does not seem to be the case to us. According to their hypothesis, false rejections and true rejections are correlated because one factor (setting of the acceptance threshold) controls both. Hence, species with high rates of false rejection should have high rates of true rejection, if the distributions for the probability of acceptance for nestmates and non-nestmates overlap or are near each other (Reeve 1989; Johnson et al. 2011). Hence, if we were to plot true and false rejections for many species (assuming each has one or more adaptive settings of the acceptance threshold and a significant portion of the species have probability distributions for acceptance of nestmates and non-nestmates that are near each other, as would have to be the case for Couvillon's work to be relevant beyond bees), then we should get a significant correlation between the two types of error, since many species would be setting their level of true and false rejections according to the same constraint. However, as we showed, false rejection errors are rare in ants, so such a pattern cannot exist. Further, as we discuss in the paper, there is an alternative mechanism for what can be underlying the verified predicted pattern in bees. This is that bees are also not rejecting their own nestmates, although it can appear that way if one conflates aggression with rejection.

The second objection is that true nestmate recognition is context dependent and only guards should be considered. This criticism ignores data on nestmate recognition and is flawed conceptually. First, Butler and Free (1952) showed that aggression against bees introduced to a foreign colony continues into the nest. Second, Breed et al. (1992) recorded rejection of non-nestmates by different groups of bees and found an average of about $30 \%$ for foragers, nurses, and others, and $55 \%$ for guards. Considering that guards number in the dozens, while the other groups number in the thousands, it is clear which group has the potential to do the most work. In short, guards are the most responsive, but they do not do this task alone. Guards can perform nestmate recognition alone, if the need for it is very small, but if large numbers of foreign workers seek entry, then the guards act as an alarm system to alert the rest of the colony to shift to nest defense.

The final criticism is that our review of the literature does not support our own model. This is incorrect because our model does not make the prediction Couvillon et al. say it does. Our model argues that overall rejection of nestmates should occur at negligible rates (or not at all), while rejection rates of non-nestmates can fluctuate depending on many factors. Hence, we should expect little or no overall rejection of nestmates across species, but highly variable rates of rejection across species for non-nestmates. If one factors in the collective explanation for what is occurring in bees, then this is the case (ignoring wasps). What we did not discuss is that the three groups (ants, wasps, and bees) are probably performing nestmate recognition in different ways, such that some species use a collective approach and others a sophisticated individual level approach. Our general conclusion, however, that rejection of nestmates is not necessary to exclude non-nestmates still holds if such a pattern exists.

\section{Conclusions}

We point out that nestmate recognition, like many other social insect traits, may sometimes be based on collective actions of positive and negative feedback, rather than a simpler individual-level process. In other words, some species may have an individual level process that is much more accurate than some models suggest, and which might preclude the need for a more complex collective process, while others may have a messier (at the individual level) collective process that, in theory, could lead to the same level of accuracy. Essentially, we argue that a rethinking of the concepts involved in nestmate recognition might be worthwhile. While we think that our model, like many first attempts at generating a shift in understanding, is simple, we think testing this model will nevertheless put us on the right track towards developing an understanding of nestmate recognition that is reflective of its true complexity.

\section{References}

Breed MD, Smith TA, Torres A (1992) Role of guard honey bee (Hymenoptera: Apidae) in nestmate discrimination and replaced of removed guards. Ann Entomol Soc Am 85:633-637

Butler CB, Free JB (1952) The behaviour of worker honey bees at the hive entrance. Behaviour 4:262-292

Couvillon MJ, Robinson EJH, Atkinson B, Child L, Dent KR, Ratnieks FLW (2008) En Garde: rapid shifts in honey bee (Apis mellifera) guarding behavior is triggered by onslaught of conspecific intruders. Anim Behav 76:225-232

Couvillon MJ, van Zweden JS, Ratnieks FLW (2011) Model of collective decision making in nestmate recognition fails to account 
for individual discriminator responses and non-independent discriminator errors. Behav Ecol Sociobiol, in press

Johnson BR, van Wilgenburg E, Tsutsui ND (2011) Nestmate recognition in social insects: overcoming physiological constraints with collective decision making. Behav Ecol Sociobiol 65:935-944

Martin S, Drijfhout F (2009) A review of ant cuticular hydrocarbons. J Chem Ecol 35:1151-1161
Reeve HK (1989) The evolution of conspecific acceptance thresholds. Am Nat 133(3):407-435

Tsutsui ND (2004) Scents of self: the expression component of self/ nonself recognition systems. Ann Zool Fenn 41:713-727

van Wilgenburg E, Clemencet J, Tsutsui ND (2010) Experience influences aggressive behaviour in the Argentine ant. Biol Lett $23: 152-155$ 